\title{
Tumor volume reduction rate is superior to RECIST for predicting the pathological response of rectal cancer treated with neoadjuvant chemoradiation: Results from a prospective study
}

\author{
JIAN XIAO $^{1 *}$, YUTING TAN $^{1 *}$, WENYUN LI ${ }^{2 *}$, JIAYING GONG $^{3}$, ZHIYANG ZHOU $^{3}$, YAN HUANG $^{4}$, \\ JIAN ZHENG ${ }^{5}$, YANHONG DENG ${ }^{1}$, LEI WANG ${ }^{6}$, JUNSHENG PENG $^{6}$, \\ DONGLIN REN ${ }^{6}$, PING LAN ${ }^{6}$ and JIANPING WANG ${ }^{6}$
}

\begin{abstract}
${ }^{1}$ Department of Medical Oncology, The Sixth Affiliated Hospital of Sun Yat-Sen University, Guangzhou, Guangdong 510655, P.R. China; ${ }^{2}$ Centre for Quantitative Medicine, Duke-NUS Graduate Medical School, National University of Singapore, Singapore 169856, Republic of Singapore; Departments of ${ }^{3}$ Radiology, ${ }^{4}$ Pathology, ${ }^{5}$ Radiation Oncology and ${ }^{6}$ Colorectal Surgery, The Sixth Affiliated Hospital of Sun Yat-Sen University, Guangzhou, Guangdong 510655, P.R. China
\end{abstract}

Received May 26, 2014; Accepted February 13, 2015

DOI: 10.3892/ol.2015.3101

\begin{abstract}
The aim of the present study was to compare the tumor volume reduction rate (TVRR), as determined by three-dimensional region-of-interest magnetic resonance volumetry, and Response Evaluation Criteria in Solid Tumors (RECIST) data for predicting the pathological tumor response (PTR) of locally advanced rectal cancer (LARC) following treatment with neoadjuvant chemoradiation (CRT). The current cohort consisted of 105 patients with LARC [clinical tumor stage (cT)3-4 or clinical lymph node stage $(\mathrm{cN})+]$ from a prospective randomized trial who had undergone pre-operative CRT and radical proctectomy. Tumor volumes were measured prior to and following CRT to determine TVRR. Furthermore, receiver operating characteristic (ROC) curves of TVRR and RECIST were constructed to predict the PTR in terms of tumor regression grade (TRG) and downstaging. Values for the area under the ROC curve (AUC) were compared and TVRR cut-off levels were determined. RECIST was used to identify $5(4.8 \%)$ cases of complete response, $44(41.9 \%)$ of partial response, $55(52.4 \%)$ of stable disease and $1(0.9 \%)$ of progressive disease. The mean TVRR was $58.6 \pm 24.4 \%$, and a good TRG (0-1) and downstaging occurred in $54(51.4 \%)$ and $59(56.2 \%)$ patients, respectively. In addition,
\end{abstract}

Correspondence to: Professor Jianping Wang, Department of Colorectal Surgery, The Sixth Affiliated Hospital of Sun Yat-Sen University, 26 Yuancun Erheng Road, Guangzhou, Guangdong 510655, P.R. China

E-mail: jianpingwangcn@126.com

"Contributed equally

Key words: rectal cancer, tumor volume reduction rate, Response Evaluation Criteria in Solid Tumors, tumor regression grade, downstaging, neoadjuvant chemoradiation
TVRR and RECIST were significantly correlated with TRG and downstaging $(\mathrm{P}<0.01)$. The TVRR AUC was significantly larger than that of RECIST for TRG ( $\mathrm{P}=0.020)$. For downstaging, TVRR also exhibited a larger AUC than RECIST, however, the difference was not significant $(\mathrm{P}=0.180)$. The sensitivity and specificity of TVRR in predicting a good TRG were 70.4 and $80.4 \%$, respectively, therefore, the optimal TVRR cut-off value was determined to be $65 \%$. TVRR appeared to be more accurate than RECIST in predicting PTR, particularly for TRG associated with survival. Thus, TVRR may be considered as a novel parameter for evaluating the efficacy of CRT for patients with LARC.

\section{Introduction}

Colorectal cancer is the third most commonly diagnosed type of cancer worldwide (1). In China, $50 \%$ of cases of colorectal cancer arise in the rectum, accounting for $>100,000$ new diagnoses of rectal cancer in 2012 (2). Despite the advent of total mesorectal excision (TME) (3) facilitating major improvements in the management of locally advanced rectal cancer (LARC), which is defined as clinical tumor stage (cT)3-4 or clinical lymph node stage $(\mathrm{cN})+$, management remains a challenge following Miles' procedure due to the high recurrence rate and poor quality of life (4).

Neoadjuvant chemoradiation (CRT) has been established as the standard treatment strategy for LARC, as it appears to be associated with improved local control and a higher rate of sphincter-sparing procedures (5). Pathological tumor response (PTR), including tumor regression grade (TRG) and downstaging, has been indicated to be an important prognostic factor for LARC following CRT (6-8). PTR may be useful to stratify patients with different prognoses and to tailor surgical treatment strategies, particularly for sphincter-sparing candidates (9). Therefore, the early and accurate prediction of PTR is important.

The modified Response Evaluation Criteria in Solid Tumors (RECIST), based on unidimensional measurements, 
is currently considered to be the gold standard for the majority of solid tumors (10). However, as a traditional diameter-based method, RECIST is unable to provide data closely reflecting the actual tumor volume change, as the CRT may induce apparent fibrosis or inflammation $(11,12)$. Additionally, the actual tumor volume change was affected by patient positioning and discrepant scan planes (13). As a canal-shaped organ, rectal cancer always presents with irregular tumor configurations and non-uniform treatment-association shrinkage to CRT $(14,15)$. Furthermore, the RECIST Working Group encourages the development of novel markers and tools to predict potential therapeutic benefit for cancer patients (16).

With the advancement in imaging techniques and their increasing availability in oncological practice, tumor volume reduction rate (TVRR), which is based on actual tumor volume change and measured using three-dimensional (3D) region-of-interest (ROI) magnetic resonance (MR) volumetry, has recently been investigated (17-19). However, to the best of our knowledge, all previous TVRR studies involved retrospective analysis, and did not perform comparisons between TVRR and RECIST.

Therefore, the present study was based on a prospective randomized trial, and was conducted to determine whether TVRR is associated with PTR in terms of TRG and downstaging. In addition, the current study aimed to determine whether TVRR is superior to RECIST in the evaluation patients with LARC following CRT.

\section{Patients and methods}

Patients. The present study is based on a prospective randomized trial (www.clinicaltrials.gov; NCT01211210). Between October 2010 and September 2013, 105 primary rectal cancer patients were treated with pre-operative CRT at the Sixth Affiliated Hospital of Sun Yat-Sen University (Guangzhou, Guangdong, China). The present study was conducted in accordance with the Declaration of Helsinki and approved by the Ethics Committee of the Sixth Affiliated Hospital of Sun Yat-Sen University. Written informed consent was obtained from all participants. The inclusion criteria were as follows: i) Histologically confirmed adenocarcinoma of the rectum; ii) distal margin of tumor located within $12 \mathrm{~cm}$ of the anal verge; iii) cT3-4 or $\mathrm{cN}+$, evaluated by $\mathrm{MR}$ imaging with or without transrectal ultrasonography; iv) no evidence of distant metastasis; v) no previous or concurrent malignancy; and vi) the availability of MR volumetry and contrast-enhanced computed tomography (CT) scan data.

Treatment strategies. The patients were treated according to the institutional protocol of Sun Yat-Sen University, as previously described (20). Pre-operative radiotherapy of $46 \mathrm{GY}$ in 23 fractions was delivered to the pelvis, followed by an optional boost of $4 \mathrm{GY}$ in two fractions to the primary tumor. All patients received 3D conformal radiotherapy with CT simulation. This three-field treatment plan included a 6-MV photon posteroanterior field and 15-MV photon opposed lateral fields.

Concurrent chemotherapy regimens included 5-fluorouracil (5-FU; Xudong Haipu Pharmaceutical Co., Ltd., Shanghai, China) alone or a doublet combination of 5-FU and
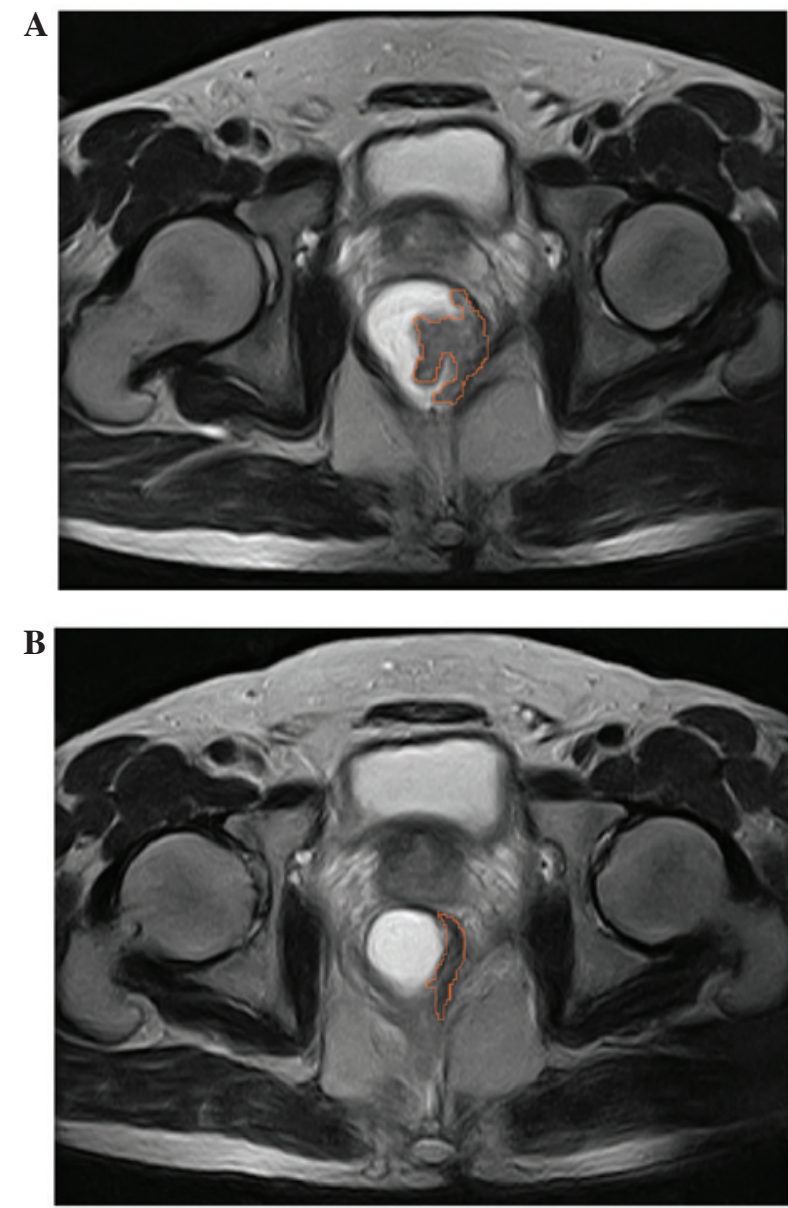

Figure 1. Axial three-dimensional region-of-interest (ROI) magnetic resonance volumetry images (A) prior to and (B) after chemoradiation. Shaded ROI represents manual tracing of the cross-sectional tumor area observed on the T2-weighted image.

oxaliplatin (Sanofi, Paris, France). 5-FU was administered according to a simplified de Gramont regimen $\left[400 \mathrm{mg} / \mathrm{m}^{2}\right.$ intravenous (i.v.) bolus followed by a 46-h protracted i.v. infusion of $2,400 \mathrm{mg} / \mathrm{m}^{2}$, every two weeks]. Oxaliplatin was administered at a dose of $85 \mathrm{mg} / \mathrm{m}^{2}$ twice weekly.

Standardized total mesorectal excision (TME) was scheduled to be performed 6-8 weeks after completion of the neoadjuvant CRT. Patients were restaged by performing a CT scan of the chest-abdomen and contrast-enhanced MR imaging of the pelvis. Creation of a temporary diverting ostomy was at the discretion of the primary surgeon, however, ostomy takedown was advised following the completion of all systemic therapy.

MR volumetry and RECIST evaluation. Two independent radiologists used identical protocol to perform 3D-ROI MR volumetry for all patients at the initial workup and within seven days of undergoing surgery (21). The cross-sectional lesion areas were measured on axial T2-weighted images by manually tracing the lesion boundaries. The contour of the cross-sectional lesions were defined as intermediate signal intensity areas that differed from the healthy adjacent rectal wall (Fig. 1). Furthermore, the tumor volumes were automatically calculated by summing each of the cross-sectional volumes (multiplying cross-sectional area by section thickness) using the Advantage 
Table I. Patient characteristics $(n=105)^{\mathrm{a}}$.

\begin{tabular}{|c|c|}
\hline Characteristic & $\mathrm{n}(\%)$ \\
\hline \multicolumn{2}{|l|}{ Gender } \\
\hline Male & $73(69.5)$ \\
\hline Female & $32(30.5)$ \\
\hline \multicolumn{2}{|c|}{ Distance from the anal verge, $\mathrm{cm}$} \\
\hline$\leq 5$ & $46(43.8)$ \\
\hline$>5$ & $59(56.2)$ \\
\hline \multicolumn{2}{|l|}{ Histological grade } \\
\hline 1 & $39(37.1)$ \\
\hline 2 & $53(50.5)$ \\
\hline 3 & $13(12.4)$ \\
\hline \multicolumn{2}{|l|}{ cT classification } \\
\hline cT2 & $2(1.9)$ \\
\hline cT3 & $84(80.0)$ \\
\hline cT4 & $19(18.1)$ \\
\hline \multicolumn{2}{|l|}{$\mathrm{cN}$ classification } \\
\hline $\mathrm{cN}-$ & $33(31.4)$ \\
\hline $\mathrm{cN}+$ & $72(68.6)$ \\
\hline \multicolumn{2}{|l|}{ Pre-CRT CEA, ng/ml } \\
\hline Normal & $75(71.4)$ \\
\hline Elevated & $30(28.6)$ \\
\hline \multicolumn{2}{|l|}{ Chemotherapy } \\
\hline 5-FU alone & $32(30.5)$ \\
\hline 5-FU plus oxaliplatin & $73(69.5)$ \\
\hline \multicolumn{2}{|l|}{ Surgical procedure } \\
\hline LAR & $71(67.6)$ \\
\hline Parks' & $20(19.0)$ \\
\hline Miles' & $14(13.4)$ \\
\hline
\end{tabular}

${ }^{a}$ The median age of the cohort was 54 years (range, 24-73 years). $\mathrm{cT}$, clinical tumor stage; $\mathrm{cN}$, clinical lymph node stage; CRT, chemoradiotherapy; CEA, carcinoembryonic antigen; LAR, low anterior resection.

Workstation (version 4.0; GE Healthcare, New York, NY, USA). The mean values determined by the two radiologists were used as the final volumetry results. In addition, TVRR was calculated as follows: TVRR $=\left(\mathrm{V}_{\text {pre-CRT }}-\mathrm{V}_{\text {post-CRT }}\right) / \mathrm{V}_{\text {pre-CRT }} \mathrm{x} 100$, where $\mathrm{V}_{\text {pre-CRT }}$ is the pre-CRT tumor volume and $\mathrm{V}_{\text {post-CRT }}$ is the post-CRT but pre-surgery tumor volume.

Positive lymph node involvement was defined as the presence of a lymph node measuring $\geq 1.0 \mathrm{~cm}$ in the smallest diameter, as observed by MR imaging. T stage was evaluated according to the method employed by Smith and Brown (22). Complete response (CR; disappearance of all target lesions), partial response $(\mathrm{PR} ; \geq 30 \%$ decrease in the sum of the diameters of the target lesions), progressive disease (PD; $\geq 20 \%$ increase in the sum of the diameters of the target lesions) and stable disease (SD; insufficient shrinkage to qualify for PR or insufficient increase to qualify for PD) states were evaluated according to RECIST, version 1.1 (16). A clinical response was defined as CR and PR.
Pathological evaluation. Following surgery, pathological evaluation was performed by an experienced pathologist, according to the tumor-node-metastasis staging system of the seventh edition of the American Joint Committee on Cancer (23). Downstaging was defined as postneoadjuvant therapy (yp) T0-2 and ypNO. Furthermore, TRG was defined using Ryan's criteria (24), as follows: Grade 0, no viable cancer cells; grade 1, single cells or small groups of cancer cells; grade 2, residual cancer outgrown by fibrosis; grade 3 , residual cancer outgrown by fibrosis or no fibrosis with extensive residual cancer. Regression grading involved the primary tumor and regional lymph nodes, with a good TRG defined as TRG grade 0 or 1 .

Statistical analysis. Continuous variables are presented as the mean \pm standard deviation or the median (range), whereas categorical variables are presented as a number (percentage). Comparisons of TVRR between independent subgroups were performed using a two-sample t-test or analysis of variance. Comparisons of RECIST between independent subgroups were performed using Fisher's Exact test. In addition, receiver operating characteristic (ROC) curves of TVRR and RECIST were constructed to predict PTR. The ROC curve was used to determine the optimal cut-off of TVRR for predicting PTR, and predictive accuracies were quantified and compared using the area under the ROC curve (AUC) (25). Sensitivities and specificities of TVRR and RECIST were also calculated. Two-sided $\mathrm{P}<0.05$ was considered to indicate a statistically significant difference, and SAS software for Windows (version 9.2; SAS Institute, Cary, NC, USA) was used for all statistical analyses.

\section{Results}

Patient characteristics. A total of 123 patients were enrolled in the present study, from which 18 patients were excluded due to withdrawal of informed consent $(n=3)$, protocol deviation $(n=3)$ or receipt of surgery elsewhere $(n=12)$. The clinical characteristics of the 105 included patients are shown in Table I. The median age was 56 years (range, 24-73 years), and the cohort consisted of $73(69.5 \%)$ male patients and $32(30.5 \%)$ female patients. A total of $84(80.0 \%)$ patients presented with cT3 disease, $72(68.6 \%)$ patients were regional lymph node-positive prior to treatment and 46 (43.8\%) patients had a low lying rectal tumor. Furthermore, combined chemotherapy was administered to 73 (69.5\%) patients. The surgical procedures performed in the present cohort included a low anterior resection (71 patients; 67.6\%), and Parks' (20 patients; 19.0\%) and Miles' (14 patients; 13.3\%) procedures.

Association of TVRR and RECIST with patient characteristics. The mean $\mathrm{V}_{\text {pre-CRT }}$ and $\mathrm{V}_{\text {post-CRT }}$ were $44.82 \pm 44.64 \mathrm{~cm}^{3}$ and $18.27 \pm 19.04 \mathrm{~cm}^{3}$, respectively, and the mean TVRR was $58.6 \pm 24.4 \%$. According to RECIST, 5 (4.8\%) patients achieved CR, 44 (41.9\%) achieved PR, 55 (52.4\%) exhibited SD and only $1(0.9 \%)$ patient experienced PD. The associations between TVRR and RECIST and the patient characteristics are presented in Table II. None of the clinical characteristics investigated were significantly associated with TVRR or RECIST. For example, patients that received the doublet chemotherapy regimen exhibited a higher mean TVRR (59.1\%) than those 
Table II. Association of patient characteristics with TVRR and RECIST.

\begin{tabular}{|c|c|c|c|c|c|}
\hline Characteristic & Patients, n (\%) & TVRR, $\%^{\mathrm{a}}$ & P-value ${ }^{b}$ & $\mathrm{CR}+\mathrm{PR}, \mathrm{n}(\%)^{\mathrm{c}}$ & P-value ${ }^{d}$ \\
\hline Gender & & & 0.379 & & 0.403 \\
\hline Male & $73(69.5)$ & $57.2 \pm 26.0$ & & $32(43.8)$ & \\
\hline Female & $32(30.5)$ & $61.8 \pm 20.1$ & & $17(53.1)$ & \\
\hline Age, years & & & 0.239 & & 0.091 \\
\hline$\leq 60$ & $72(68.6)$ & $58.2 \pm 20.5$ & & $38(52.8)$ & \\
\hline$>60$ & $33(31.4)$ & $53.6 \pm 29.2$ & & $11(33.3)$ & \\
\hline Distance from the anal verge, $\mathrm{cm}$ & & & 0.774 & & 0.237 \\
\hline$\leq 5$ & $46(43.8)$ & $57.9 \pm 24.2$ & & $18(39.1)$ & \\
\hline$>5$ & $59(56.2)$ & $59.2 \pm 24.7$ & & $31(52.5)$ & \\
\hline Histological grade & & & 0.652 & & 0.127 \\
\hline 1 & $39(37.1)$ & $60.5 \pm 20.4$ & & $23(59.0)$ & \\
\hline 2 & $53(50.5)$ & $58.6 \pm 28.4$ & & $22(41.5)$ & \\
\hline 3 & $13(12.4)$ & $53.2 \pm 17.3$ & & $4(30.8)$ & \\
\hline Clinical T classification & & & 0.596 & & 0.317 \\
\hline cT2-T3 & $86(81.9)$ & $58.2 \pm 26.1$ & & $38(44.2)$ & \\
\hline $\mathrm{cT} 4$ & $19(18.1)$ & $60.6 \pm 14.7$ & & $11(57.9)$ & \\
\hline Clinical N classification & & & 0.936 & & 0.346 \\
\hline $\mathrm{cN}-$ & $33(31.4)$ & $58.3 \pm 20.9$ & & $15(45.5)$ & \\
\hline $\mathrm{cN}+$ & $72(68.6)$ & $58.8 \pm 26.0$ & & $34(47.2)$ & \\
\hline Pre-CRT CEA, ng/ml & & & 0.589 & & 0.516 \\
\hline Normal & $75(71.4)$ & $59.5 \pm 24.9$ & & $37(49.3)$ & \\
\hline Elevated & $30(28.6)$ & $56.6 \pm 23.4$ & & $12(40 \%)$ & \\
\hline Treatment group & & & 0.766 & & 0.403 \\
\hline 5-FU alone & $32(30.5)$ & $57.6 \pm 20.3$ & & $17(53.1)$ & \\
\hline 5-FU plus oxaliplatin & $73(69.5)$ & $59.1 \pm 26.1$ & & $32(43.8)$ & \\
\hline
\end{tabular}

${ }^{a}$ TVRR presented as mean \pm standard deviation. P-values were determined using btwo-sample t-test or analysis of variance and ${ }^{\mathrm{d}}$ Fisher's Exact test. 'As determined by RECIST. TVRR, tumor volume reduction rate; RECIST, Response Evaluation Criteria in Solid Tumors; CRT, chemoradiotherapy, CEA, carcinoembryonic antigen.

Table III. Association of TVRR and RECIST with PTR.

\begin{tabular}{lccc}
\hline Response & TVRR, $\%^{\mathrm{a}}$ & P-value $^{\mathrm{b}}$ & CR + PR, n $(\%)^{\mathrm{c}}$ \\
\hline TRG & & $<0.001$ & 0.001 \\
$0-1$ & $70.2 \pm 15.2$ & & $34(63.0)$ \\
$2-3$ & $46.4 \pm 26.4$ & $<(29.4)$ \\
Downstaging & & $<0.001$ & $35(58.3)$ \\
Yes & $66.5 \pm 18.0$ & & $14(31.1)$ \\
No & $48.2 \pm 27.9$ & & 0.006 \\
\hline
\end{tabular}

${ }^{\mathrm{a}}$ TVRR presented as mean \pm standard deviation. P-values were determined using ${ }^{\mathrm{b} a}$ two-sample t-test or analysis of variance and ${ }^{\mathrm{d}} \mathrm{Fisher}$ 's Exact test. 'As determined by RECIST. TVRR, tumor volume reduction rate; RECIST, Response Evaluation Criteria in Solid Tumors; PTR, pathological tumor response; TRG, tumor regression grade.

treated with single-agent chemotherapy (57.6\%); however, the difference was not statistically significant $(\mathrm{P}=0.766)$.

Association of TVRR and RECIST with PTR. Following CRT and pathological evaluation, TRG 0,1,2 and 3 was identified in $12(11.5 \%), 42(40.0 \%), 31(29.5 \%)$ and 20 (19.0\%) patients, respectively. Additionally, $59(56.2 \%)$ patients achieved downstaging. Table III shows the TVRRs and RECIST values according to the PTR findings. The TVRRs were significantly higher among patients with good TRG (70.2 vs. 46.4\%; 
Table IV. Predictive values of TVRR and RECIST for PTR.

\begin{tabular}{lrr}
\hline Response & TVRR, \% (95\% CI) & RECIST, \% (95\% CI) \\
\hline Downstaging & & $58.3(44.9-70.9)$ \\
Sensitivity & $61.7(48.2-73.9)$ & $68.9(53.4-81.8)$ \\
Specificity & $75.6(60.5-87.1)$ & \\
TRG & $70.4(56.4-82.0)$ & $63.0(48.7-75.7)$ \\
Sensitivity & $80.4(66.9-90.2)$ & $70.6(56.2-82.5)$ \\
Specificity & & \\
\hline
\end{tabular}

TVRR, tumor volume reduction rate; RECIST, Response Evaluation Criteria in Solid Tumors; PTR, pathological tumor response; TRG, tumor regression grade; $\mathrm{CI}$, confidence interval.
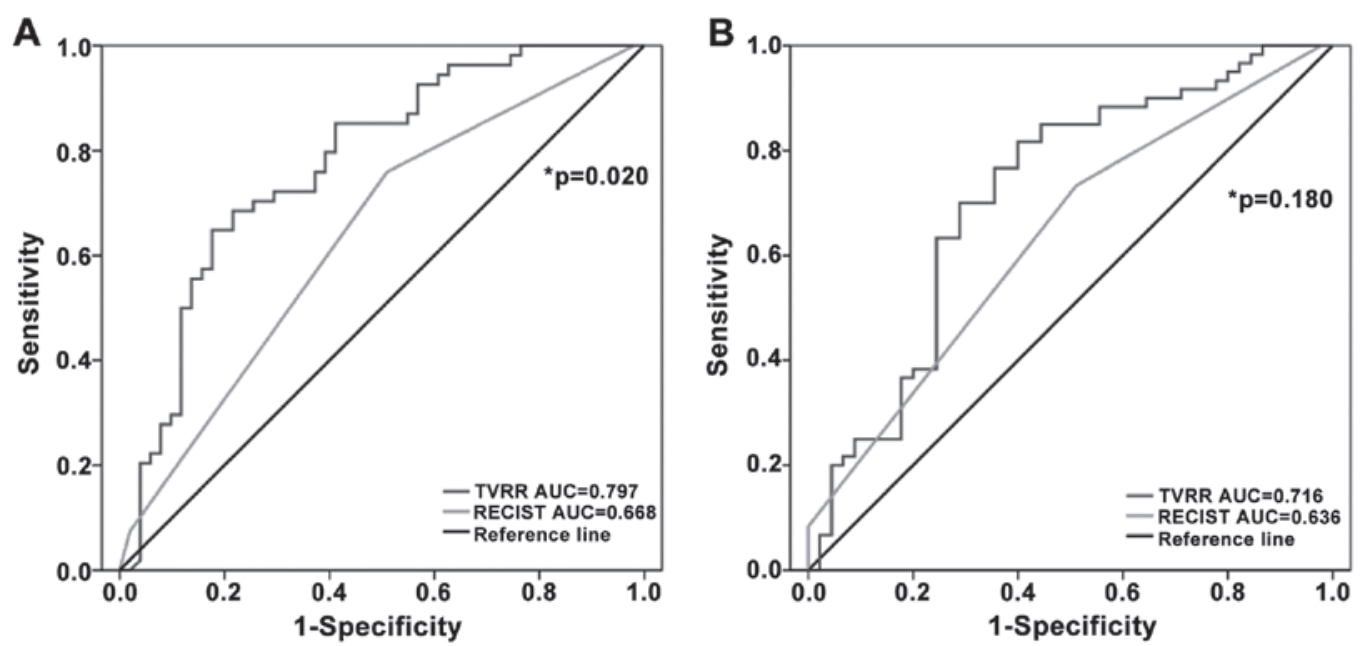

Figure 2. Receiver operating characteristic curves of TVRR and RECIST. The difference in the AUC was (A) significant for the tumor regression grade $(\mathrm{P}=0.020)$ but $(\mathrm{B})$ not significant for downstaging $(\mathrm{P}=0.180)$. TVRR, tumor volume reduction rate; RECIST, Response Evaluation Criteria in Solid Tumors; AUC, area under the curve.

$\mathrm{P}<0.001)$. Additionally, patients with downstaging exhibited significantly higher TVRRs compared with those without downstaging (66.5 vs. 48.2\%; $\mathrm{P}<0.001$ ). For RECIST, a clinical response was more frequently observed in patients with good TRG and downstaging ( $\mathrm{P}=0.001$ and $\mathrm{P}=0.006$, respectively).

Predictive values of TVRR and RECIST for PTR. ROC curves of TVRR and RECIST were constructed and compared (Fig. 2). For the prediction of TRG, the AUC of TVRR was significantly larger than that of RECIST (TVRR AUC, 0.797; 95\% CI, 0.710-0.885; vs. RECIST AUC, 0.668; 95\% CI, 0.577-0.758; P=0.020]. Similarly, the AUC of TVRR was greater than that of RECIST for downstaging (TVRR AUC, 0.716; 95\% CI, 0.612-0.819; vs. RECIST AUC, 0.636; 95\% CI, 0.543-0.729); however, the difference did not reach statistical significance $(\mathrm{P}=0.180)$.

The optimum cut-off for TVRR was determined to be $65 \%$ by a trade-off between sensitivity and specificity, thus, $\geq 65 \%$ TVRR was considered to indicate a clinical response. Using this optimal cut-off value, TVRR attained higher predictive values compared with RECIST (Table IV). For TRG, the sensitivity and specificity of TVRR were 70.4 (95\% CI, 56.4-82.0\%) and 80.4\% (95\% CI, 66.9-90.2), respectively. For downstaging, the sensitivity and specificity of TVRR were 61.7 (95\% CI, 48.2-73.9) and 75.6\% (95\% CI, 60.5-87.1), respectively.

\section{Discussion}

In the present study, the data of 105 cases of LARC from a prospective randomized trial were analyzed. Compared with RECIST, TVRR appeared to be a superior method for predicting PTR, particularly TRG, of LARC patients who had received CRT.

At present, low lying rectal cancers are associated with relatively high local recurrence rates and a poor quality of life following sphincter ablation $(4,26)$. In the current cohort, all patients, excluding one, received complete resection and $91(86.7 \%)$ patients underwent sphincter-preserving surgery. These results appear to be an improvement on previous reports $(3,5,27)$. The high sphincter-preserving rate observed in the present study may be associated with the fact that the majority of patients underwent a novel treatment strategy termed two-stage TME (20). The PTRs of patients in the present study were consistent with a previous study by Fokas et al (9); a good TRG was determined in 54 (51.4\%) 
patients and downstaging was observed in $59(56.2 \%)$ patients, indicating that the novel strategy of two-stage TME was safe and effective.

In the present study, 3D ROI MR volumetry was used to determine TVRR. The mean $\mathrm{V}_{\text {pre-CRT }}$ was $44.82 \pm 44.64 \mathrm{~cm}^{3}$ and the mean $\mathrm{V}_{\text {post-CRT }}$ was $18.27 \pm 19.04 \mathrm{~cm}^{3}$. Previous studies $(14,15,17)$ have reported a mean $\mathrm{V}_{\text {pre-CRT }}$ range of $19.0-58.0 \mathrm{~cm}^{3}$ and a mean $\mathrm{V}_{\text {post-CRT }}$ range of $6.0-20.0 \mathrm{~cm}^{3}$. This implies that 3D ROI MR volumetry is a reliable and reproducible method in clinical practice, and may be suitable for wide application with the availability of MR and a computer station.

The results of the present study identified no significant association between patient demographics and TVRR and RECIST. In agreement, a previously conducted large-scale retrospective study (28) identified no significant associations, and demonstrated that TVRR and RECIST were independent clinical methods for assessing treatment efficacy. Furthermore, TVRR and RECIST were significantly associated with PTR, including TRG, and downstaging in the current analysis. This is consistent with previous studies $(17,18,28,29)$, and confirms that the two parameters are effective and reliable.

To the best of our knowledge, the present data indicates for the first time that volumetric-based TVRR is more accurate than diameter-based RECIST in predicting a good TRG. In agreement with findings from previous studies, which ranged from 45 to $70 \%(17,18,28)$, a TVRR cut-off value of $65 \%$ was selected in the present study. All previous studies were retrospective, while the current data was prospectively collected. Therefore, the current results should be carefully interpreted and the cut-off value of $65 \%$ should be assessed by additional follow-up, including long-term outcome. Volumetric measurement requires more labor and time compared with diameter measurement. However, in the present study, each rectal volumetric examination took $\sim 15$ min compared with the $30 \mathrm{~min}$ reported by Nougaret et al (17). Furthermore, semi-automated volumetry using 3D MR, which appears to be more feasible, accurate and reproducible, requires additional investigation.

Valentini et al (8) reported that patients that downstaged (ypT0-T2) following pre-operative CRT exhibited five-year local control rates of $83-100 \%$ and overall survival rates of $81-91 \%$. These rates are similar to those of cT1-T2 patients treated with conservative surgery alone. Nougaret et al (17) reported that patients with a TVRR of $\geq 70 \%$ exhibited significantly longer DFS times (hazard ratio, 13.70; 95\% $\mathrm{CI}, 3.98-31.93 ; \mathrm{P}<0.001)$ and that TVRR was an independent prognostic parameter for DFS $(\mathrm{P}=0.003)$. However, the overall survival and relapse-free survival data of the present study have yet to mature. Therefore, this data require analysis at a later date to clarify the preliminary results.

In conclusion, the present analysis compared TVRR determined by 3D ROI MR volumetry with RECIST for predicting PTR following CRT and demonstrated the superiority of TVRR. The TVRR cut-off value of $65 \%$ is a parameter that can easily be used as a surrogate for clinical response to predict TRG. Thus, confirmation of TVRR as a parameter for predicting PTR and long-term outcome in rectal cancer is warranted.

\section{References}

1. Siegel R, Naishadham D and Jemal A: Cancer statistics, 2013. CA Cancer J Clin 63: 11-30, 2013.

2. Chen W, Zheng R, Zhang S, et al: Annual report on status of cancer in China, 2010. Chin J Cancer Res 26: 48-58, 2014.

3. Maurer CA, Renzulli P, Kull C, et al: The impact of the introduction of total mesorectal excision on local recurrence rate and survival in rectal cancer: long-term results. Ann Surg Oncol 18: 1899-1906, 2011.

4. Wiltink LM, Chen TY, Nout RA, et al: Health-related quality of life 14 years after preoperative short-term radiotherapy and total mesorectal excision for rectal cancer: report of a multicenter randomised trial. Eur J Cancer 50: 2390-2398, 2014.

5. Sauer R, Liersch T, Merkel S, et al: Preoperative versus postoperative chemoradiotherapy for locally advanced rectal cancer: results of the German CAO/ARO/AIO-94 randomized phase III trial after a median follow-up of 11 years. J Clin Oncol 30: 1926-1933, 2012.

6. Sannier A, Lefèvre JH, Panis Y, et al: Pathological prognostic factors in locally advanced rectal carcinoma after neoadjuvant radiochemotherapy: analysis of 113 cases. Histopathology 65: 623-630, 2014.

7. Dhadda AS, Bessell EM, Scholefield J, et al: Mandard tumour regression grade, perineural invasion, circumferential resection margin and post-chemoradiation nodal status strongly predict outcome in locally advanced rectal cancer treated with preoperative chemoradiotherapy. Clin Oncol (R Coll Radiol) 26: 197-202, 2014.

8. Valentini V, Coco C, Picciocchi A, et al: Does downstaging predict improved outcome after preoperative chemoradiation for extraperitoneal locally advanced rectal cancer? A long-term analysis of 165 patients. Int J Radiat Oncol Biol Phys 53: 664-674, 2002.

9. Fokas E, Liersch T, Fietkau R, et al: Tumor regression grading after preoperative chemoradiotherapy for locally advanced rectal carcinoma revisited: updated results of the CAO/ARO/AIO-94 Trial. J Clin Oncol 32: 1554-1562, 2014.

10. Therasse P, Arbuck SG, Eisenhauer EA, et al: New guidelines to evaluate the response to treatment in solid tumors. European Organization for Research and Treatment of Cancer, National Cancer Institute of the United States, National Cancer Institute of Canada. J Natl Cancer Inst 92: 205-216, 2000.

11. Curvo-Semedo L, Lambregts DM, Maas M, et al: Rectal cancer: assessment of complete response to preoperative combined radiation therapy with chemotherapy-conventional MR volumetry versus diffusion-weighted MR imaging. Radiology 260: 734-743, 2011.

12. Chen CC, Lee RC, Lin JK, et al: How accurate is magnetic resonance imaging in restaging rectal cancer in patients receiving preoperative combined chemoradiotherapy. Dis Colon Rectum 48: 722-728, 2005.

13. Wang JZ, Mayr NA, Zhang D, et al: Sequential magnetic resonance imaging of cervical cancer: the predictive value of absolute tumor volume and regression ratio measured before, during, and after radiation therapy. Cancer 116: 5093-5101, 2010.

14. Torkzad M, Lindholm J, Martling A and Blomqvist L: Retrospective measurement of different size parameters of nonradiated rectal cancer on MR images and pathology slides and their comparison. Eur Radiol 13: 2271-2277, 2003.

15. Mayr NA, Yuh WT, Taoka T, et al: Serial therapy-induced changes in tumor shape in cervical cancer and their impact on assessing tumor volume and treatment response. AJR Am J Roentgenol 187: 65-72, 2006.

16. Kang JH, Kim YC, Kim H, et al: Tumor volume changes assessed by three-dimensional magnetic resonance volumetry in rectal cancer patients after preoperative chemoradiation: the impact of the volume reduction ratio on the prediction of pathologic complete response. Int J Radiat Oncol Biol Phys 76: 1018-1025, 2010 .

17. Nougaret S, Rouanet P, Molinari N, et al: MR volumetric measurement of low rectal cancer helps predict tumor response and outcome after combined chemotherapy and radiation therapy. Radiology 263: 409-418, 2012.

18. Yeo SG, Kim DY, Park JW, et al: Tumor volume reduction rate after preoperative chemoradiotherapy as a prognostic factor in locally advanced rectal cancer. Int J Radiat Oncol Biol Phys 82: e193-e199, 2012. 
19. Wang T, Wang J, Deng Y, Wu X and Wang L: Neoadjuvant therapy followed by local excision and two-stage total mesorectal excision: a new strategy for sphincter preservation in locally advanced ultra-low rectal cancer. Gastroenterol Rep (Oxf) 2: 37-43, 2014.

20. Barbaro B, Vitale R, Leccisotti L, et al: Restaging locally advanced rectal cancer with MR imaging after chemoradiation therapy. Radiographics 30: 699-716, 2010.

21. Smith $\mathrm{N}$ and Brown G: Preoperative staging of rectal cancer. Acta Oncol 47: 20-31, 2008.

22. Eisenhauer EA, Therasse P, Bogaerts J, et al: New response evaluation criteria in solid tumours: revised RECIST guideline (version 1.1). Eur J Cancer 45: 228-247, 2009.

23. Sobin LH and Compton CC: TNM seventh edition: what's new, what's changed: communication from the International Union Against Cancer and the American Joint Committee on Cancer. Cancer 116: 5336-5339, 2010.

24. Ryan R, Gibbons D, Hyland JM, et al: Pathological response following long-course neoadjuvant chemoradiotherapy for locally advanced rectal cancer. Histopathology 47: 141-146, 2005.
25. DeLong ER, DeLong DM and Clarke-Pearson DL: Comparing the areas under two or more correlated receiver operating characteristic curves: a nonparametric approach. Biometrics 44: 837-845, 1988 .

26. den Dulk M, Putter H, Collette L, et al: The abdominoperineal resection itself is associated with an adverse outcome: the European experience based on a pooled analysis of five European randomised clinical trials on rectal cancer. Eur J Cancer 45: 1175-1183, 2009.

27. Bosset JF, Collette L, Calais G, et al: Chemotherapy with preoperative radiotherapy in rectal cancer. N Engl J Med 355: 1114-1123, 2006.

28. Yeo SG, Kim DY, Kim TH, et al: Tumor volume reduction rate measured by magnetic resonance volumetry correlated with pathologic tumor response of preoperative chemoradiotherapy for rectal cancer. Int J Radiat Oncol Biol Phys 78: 164-171, 2010.

29. Park IJ, You YN, Agarwal A, et al: Neoadjuvant treatment response as an early response indicator for patients with rectal cancer. J Clin Oncol 30: 1770-1776, 2012. 